\title{
Adherence to Kinect Adventure Games in Individuals with Parkinson's Disease - An Experimental Study from Mysore, India
}

\author{
Thillai Vignesh ${ }^{1}$, Renukadevi Mahadevan², Sandeep Padantaya Harish ${ }^{3}$ \\ ${ }^{1}$ Department of Physiotherapy, Cauvery College of Physiotherapy, Mysore, Karnataka, India, \\ 2,3 Department of Physiotherapy, JSS College of Physiotherapy, MG Road, Mysore, Karnataka, India.
}

\section{ABSTRACT}

\section{BACKGROUND}

Individuals with Parkinson's disease (PD) experience reduced balance ability and are prone to fall. As the disease advances with age, the traditional forms of physiotherapy are prone to fail in long-term exercise intervention as patients lose interest in performing the exercises. The use of virtual reality game technology consists of tasks in virtual environments that combine physical and cognitive demands in a fun and motivating manner which leads to adherence in a long-term exercise intervention. The objectives of the study were to know the adherence of Kinect adventure games in individuals with PD by attendance percentage of prescribed sessions and analyse the impact of balance after the games in them.

\section{METHODS}

An experimental study with pre-post design in which 11 individuals were selected, with a mean age of 67.8 years. Home-based balance exercises were given three days a week up to 6 weeks (18 sessions). Kinect adventure games were implemented for another six weeks. Adherence was measured by attendance percentage in terms of the frequency of sessions completed. The secondary outcome measure was to assess balance. Participants were evaluated before intervention and post-intervention using BESTest. Descriptive statistics were used to compare the BESTest scores of pre and post-intervention.

\section{RESULTS}

The adherence percentage of Kinect adventure game therapy was $38 \%$. The adherence percentage of home-based balance exercises was only $9 \%$. The best scores improved in home-based balance exercise compared to Kinect adventure game therapy.

\section{CONCLUSIONS}

The participants with Parkinson's disease did not adhere to Kinect adventure game therapy. For the attended sessions the balance improved for home-based balance exercise compared to Kinect game therapy.

\section{KEY WORDS}

Exercise Barrier, Adherence, Balance Training, Virtual Reality, Video Games
Corresponding Author: Dr. Renukadevi Mahadevan, Professor,

Department of Physiotherapy, JSS College of Physiotherapy, MG Road, Mysore, Karnataka, India. E-mail: renukaramya01@gmail.com

\section{DOI: $10.14260 /$ jemds/2021/562}

How to Cite This Article:

Vignesh T, Mahadevan $R$, Harish SP. Adherence to kinect adventure games in individuals with Parkinson's disease - an experimental study from Mysore, India. J Evolution Med Dent Sci 2021;10(33): 2754-2760, DOI:

$10.14260 / \mathrm{jemds} / 2021 / 562$

Submission 28-11-2020,

Peer Review 29-05-2021,

Acceptance 05-06-2021,

Published 16-08-2021.

Copyright (C) 2021 Thillai Vignesh et al. This is an open access article distributed under Creative Commons Attribution License [Attribution 4.0 International (CC $B Y 4.0)]$ 


\section{BACKGROUND}

Parkinson's disease (PD) can be defined as a neurodegenerative disorder that causes progressive motor symptoms such as tremor, rigidity, bradykinesia, gait instability, balance dysfunction, and cognitive deficits leading to limitations across various domains of the International classification of functioning, disability and health (ICF), that is body function, activity limitation, participation restriction. ${ }^{1}$ It is estimated that approximately about 60 lakh individuals suffer from PD worldwide. World Health Organisation (WHO) reports that, out of the world's 580 million elderly (over 60 years) population, 16 - 19 per lakh suffer from PD, of which $60 \%$ live in developing countries and $22 \%$ live in India.

The basal ganglia structures involved in the disease process are neostriatum, external and internal pallidal segments, subthalamic nucleus, and substantia nigra, which are responsible for the smooth and purposeful coordination of body movements. ${ }^{2}$ In PD, there is degeneration of dopaminergic neurons and their extensions to the striatum. Substantia nigra projections to the putamen degenerate earlier and during this degeneration, motor symptoms and signs of PD appear. These symptoms occur only following the substantial degeneration of the nigrostriatal neurons, which affects at least $70 \% .^{3}$ The pathophysiology of balance impairments in PD includes various subsystems which involve sensory, motor, and cognition. Sensory issues compromise equilibrium, which leads to impaired proprioception \& motor features with cognitive deficiencies further worsening the efficiency of balance control. ${ }^{4}$ The commonly used drugs for managing Parkinson's disease are levodopa, MAO - B inhibitors, a dopamine agonist, anticholinergic agents, amantadine. ${ }^{5}$ Even though pharmacological interventions were able to alter the natural course of the disease, they cannot reverse the balance dysfunction and the cardinal features of PD. These symptoms exacerbate over time as the disease advances with a significant deterioration of the patient's exercise capacity and quality of life.

Microsoft Game Studios developed Kinect Adventure games for Xbox 360. It is a sports video game allowing the entire body movement to involve the participant in multiple mini-games to attain the maximum number of points. The adventure points which are earned are represented in medals that influence the game progression. The medals are classified into bronze, silver, gold and platinum, in order of least to maximum pins needed to acquire in each game. ${ }^{6}$

World Health Organization (WHO) defines adherence as the extent to which a person's behaviour following a diet, taking medication and / or executing lifestyle changescorresponds to the recommendations of a healthcare provider. Exercise adherence can be defined as the degree to which a patient complies with the advised interval and exercise dosing regimen. The unit of measure for adherence is measured as performed exercise doses, per defined period reported as a proportion of prescribed exercise doses undergone at the prescribed time interval. Patients can be separated into 3 groups based on their adherence to the exercise protocol that was prescribed. The $1^{\text {st }}$ group is patients who adhere at least $80 \%$ to the number of training sessions prescribed and to the duration of the prescribed cycle. The $2^{\text {nd }}$ group consists of patients who are adherent less than $20 \%$ to the advised number of training sessions \& their duration. The $3^{\text {rd }}$ group consists of partially adherent patients who perform the prescribed exercises, but avoid some of the exercises or they will not perform them for the prescribed duration ${ }^{7}$

A cross-sectional study was done by Miller $\mathrm{K}$ et al. states that $65.3 \%$ reported adherence to a home exercise programme within the first 6 months post-discharge. The adherence rates for exercises have been reported in elderly individuals with arthritis, low back pain (LBP) and a general physical therapy population ranging from 40 to $71 \%$. The reason for non-adherence documented in that study was doing exercises other than what was prescribed by the physiotherapist as the exercise was painful, boring and fear of fall while exercising. 8

A systematic review done by Yitayeh et al. states that there is a short-term benefit in enhancing balance and mobility in individuals with PD using physiotherapy interventions like balance training, strength training and aerobic training. Conventional physiotherapies involved in the management of Parkinson's disease are cognitive movement strategies, cueing strategies, balance training, gait training, aerobic training, relaxation techniques, strengthening exercises and flexibility training.

The use of virtual reality game technology such as (Sony play station, Nintendo Wii, Kinect etc.) are rapidly increasing in neurological rehabilitation. ${ }^{7}$ Virtual reality games consist of tasks in virtual environments which combine physical and cognitive demands in a way that is attractive and challenging. ${ }^{7}$ The player is motivated to attain goals and overcome his / her limits during the games, which provide immediate feedback on his / her performance. In addition, virtual reality games like Kinect adventures engage patients in executing simple or complex exercise patterns within a goal-oriented enjoyable context, with visual and auditory feedback.

Regular physiotherapy has a major role in controlling symptoms in PD. As the disease progresses with age the traditional forms of physiotherapy are prone to fail in long term rehabilitation as patients lose interest in performing the same form of tasks and exercises day after day and get easily bored and eventually drop out of a rehabilitation programme.7,8 It is well documented that, due to lack of interest, fear of falling and the progressive nature of the disease, there is a lack of adherence to exercises which becomes the barrier to short-term or long-term exercise intervention in chronic conditions that need the exercise as an intervention. This leads to poor outcomes.

Kinect adventure games are a set of games that involve whole-body motion that is challenging, stimulate users to exercise as there are rewards and scores which would be fun and motivating, therefore the adherence to the exercise would enhance and thereby improves the balance. Therefore, the need of this study was to know the adherence of Kinect adventure games in patients with Parkinson's disease. 


\section{METHODS}

The study design was an experimental type (Pre \& Post design) and the source of the study was Basal ganglia society, JSS Hospital Neuro Department, Mysuru. The sampling technique was convenience sampling, and the sample size was a complete enumeration of participants (within 6 months of the time -period).

\section{Inclusion Criteria}

Patients diagnosed with Parkinson's disease of stages 1,2 \& 3 of Hoehn \& Yahrscale, patients with mini-mental state examination score $\geq 20$ and patients with Berg Balance Scale score $\geq 46$.

\section{Exclusion Criteria}

Haemodynamically unstable patients, patients with unstable angina, patients with recent myocardial infarction, patients with any history of seizure, patients involved in other clinical trials, patients involved in a trial of the investigational drug, patients with orthopaedic or neurological associated diseases, patients with visual impairment and patients with auditory impairment.

\section{Procedure}

Ethical clearance was obtained from the institutional ethical committee of JSS medical college (Ethical approval letter number-(JSSMC/IEC/3107/16 NCT). There was a total of 110 patients registered in Basal Ganglia society in Mysuru, with permission the complete list of contact information was obtained, and the participants were recruited. The participants were approached by phone and a prior appointment was taken to visit their homes. Explanation about the needs, procedure and benefits of the exercise programme was explained to the participants. Informed consent was taken from the participants who were willing to participate in the study. Then the participants were enrolled based on the inclusion and exclusion criteria. A pilot study was done in JSS PMRC, Mysuru and three PD patients were implemented in this study. To standardize the procedure of the study and the pilot study objectives were to examine the safety of the participants, the feasibility of the games, interest and standardization of the procedure before a large trial was attempted. The participants were asked to play the games by standing in front of a "32" TV at a minimum distance of $3 \mathrm{~m}$.

The Xbox 360 videogame console and Kinect sensor were fixed in a manner so that participants will be viewed properly, and the Kinect setup had infrared sensors which capture the player's movements through a camera. An avatar was reproduced in the virtual environment from the movement of the player captured by the camera. Then the participants were asked to play a Kinect adventure game which consisted of Space Pop, 20,000 Leaks, Reflex Ridge and River Rush for three repetitions where each game was of 2 minutes followed by a rest period of 4 minutes where a chair was given for the patient to sit. The session lasted for about 60 minutes. The results of the pilot study indicated that the River Rush was one of the four - games that were found to be highly challenging and required more repetitive fast movement with multiple jumps, so participants found it difficult to explore the game and another important factor found after the game was fatigue. From the results based on feasibility and safety, the River Rush game was removed and a set of four games were reduced to three games.

The main study was initiated with a baseline score of BESTest which was taken before the intervention. The study duration was from August 2018 to January 2019. All recruited participants were given handouts which consisted of a home-based balance exercise with pictures and descriptions in English and Kannada for 3 days per week up to six weeks (18 sessions) and each session lasted for 60 minutes 16. Adherence to the exercise was assessed from the logbook which comprised the date, number of sessions, and number of repetitions and duration of the exercise. Documentation of the frequency of exercises in the daily logbook was explained to the participants and the caregivers. The participants' homes were visited at the end of each week and their logbooks were checked. Doubts and clarification about the exercises presented by the participants were reeducated. At the end of the sixth week, the percentage of attendance for the home-based balance exercise was calculated from the logbook in terms of the frequency of the exercise performed. The baseline value to check adherence was kept at $75 \%$. The participants who had $\geq 75 \%$ of attendance were considered as the ones adhering to the intervention. The reason for non-adherence was documented by the researcher. Home-based balance training consists of exercises: 1) Standing equal to shoulder - width, feet together, 2) Tandem standing, Partial Tandem standing, 3) Standing in one leg, 4) Weight - shift (Forward, backward, both the sides), 5) Sternal Perturbations from the front, Dorsal Perturbations from back, 6) Standing on toes, standing on heels, 7) Catching the ball from the front, back, both sides, half - squat. All the exercises should be performed with eyes open for 1-3 weeks and eyes closed for $4-6$ weeks. $^{9-14}$

After 6 weeks of home-based balance exercises, BESTest was evaluated to know the improvement in balance which was considered as baseline BESTest score for Kinect game therapy. Then the participants were called back to JSS Physical Medicine and Rehabilitation Centre (PMR), Mysuru for the intervention. Demonstration and trial of Kinect adventure games were given to get familiarized by the participants and the procedure of gaming was explained to the participants and caregivers by the researcher. They underwent 3 rounds of all three games which lasted for an hour with a rest period of $4 \mathrm{~min}$ between each round. During the rest period, the subjects were informed to sit in the chair. ${ }^{15}$ Throughout the first two sessions, verbal comments were given to participants to perform actions that were needed to explore the game and encourage the right posture. A harness or gait belt was given for the participants based on their balance status. Participants were asked to inform the researcher if any problem or discomfort occurred during the session.

After each game, the scores obtained were displayed on the monitor and medals were given as a reward to perform better for the next session. Adherence to the game was calculated based on a session documented in the rehabilitation centre using logbook BESTest and after the intervention of 12 weeks, post-intervention was evaluated. 


\section{Statistical Analysis}

Data analysis of Kinect game therapy and home-based balance exercise adherence was calculated by descriptive statistics Data analysis of pre and post-test BESTest was calculated by paired " $\mathrm{t}$ " test.

\section{RESULTS}

A total of 11 Parkinson's disease patients were enrolled for the study ( 8 men, 3 women) with a mean age of 67.8 years (SD of 7.54). Demographic characteristics of the participants are presented in Table 1. All participants' data was entered initially through an excel sheet and using descriptive statistics, analysis for adherence and the pre-test and posttest balance scores were calculated in terms of percentage. All the participants recruited for the study were included for analysis. BESTest was evaluated in the baseline, 6 weeks after home-based balance exercises and 12 weeks after Kinect adventure game therapy.

\begin{tabular}{|cc|}
\hline Demographics & Group \\
Age (years) & $67.81 \pm 7.54$ \\
Weight (kg) & $66.63 \pm 3.07$ \\
Chronicity (years) & $6.18 \pm 2.44$ \\
BBS & $48.36 \pm 2.65$ \\
MMSE & $28.45 \pm 1.29$ \\
\hline Table 1. Participants Demographic Characteristics \\
\hline
\end{tabular}

\section{Primary Outcome Measure - Adherence to Home-Based Balance Exercises}

Adherence to home-based balance exercises was calculated by attendance in terms of the frequency of the exercise performed by the participants.

\section{Frequency of the Exercise}

All participants recruited for the study were analysed for adherence. The frequency of the exercise was calculated in terms of the repetition of the exercises that the participants performed, and values were entered in terms of percentage.

Descriptive statistics were used to find the mean percentage frequency of exercise for each participant. Attendance was categorized based on the percentage obtained. Attendance of seventy-five per cent and above was considered as adherence. The analysis showed that only one patient was adherent to home-based balance training which was $9 \%$, which indicated that participants were nonadherent to the home-based balance exercises.

As shown in Table 2, out of 11 participants, five participants adherence was less than $25 \%$, two participants adherence was between $26-50 \%$, three participants adherence was between 51 - $75 \%$ which indicates that these participants were non-adherent to the home-based balance exercise and only one patient adherence was more than $75 \%$ which indicates that participant was adherent to home-based balance exercises. The overall percentage of adherence to home-based balance exercise was only $9 \%$.

\begin{tabular}{|c|c|c|c|}
\hline Adherent & Number of & \multicolumn{2}{|c|}{ Range of BESTest Range of BESTest } \\
\hline Percentage & Participants & in Baseline & in Post-Test \\
\hline$<25 \%$ & $5 / 11$ & $67-78$ & $77-79$ \\
\hline $26-50 \%$ & $2 / 11$ & $80-86$ & $85-90$ \\
\hline $51-75 \%$ & $3 / 11$ & $65-74$ & $63-80$ \\
\hline $76-100 \%$ & $1 / 11$ & 82 & 92 \\
\hline \multicolumn{4}{|c|}{$\begin{array}{l}\text { Table 2. The Adherence Percentage, No of Participants and Range of } \\
\text { Bestest Scores in Pre and Post Intervention of Home-Based Balance } \\
\text { Exercises }(N=11)\end{array}$} \\
\hline
\end{tabular}

\section{Secondary Outcome Measure - Bestest}

The difference between pre-test and post-test home-based balance training was analysed with Paired t-test

$H_{0}$ : mean effects of pre-test and post-test home balance training was equal.

$H_{1}$ : mean effect of post-test home balance training was greater than pre-test training.

\begin{tabular}{|ccc|}
\hline $\mathbf{t}$ & df & P-Value \\
\hline 2.8786 & 10 & 0.008212 \\
\hline Table3. The Difference between Pre and Post-Test Balance Scores of \\
\multicolumn{3}{|c|}{ Home-Based Balance Training. } \\
\hline
\end{tabular}

Since the P-value was less than 0.05 , there is strong evidence to reject the null hypotheses. Hence mean effect of post-test home balance training was greater than pre-test training.

\section{Adherence to Kinect Adventures Game Therapy}

Adherence to Kinect adventure game therapy was calculated by attendance in terms of the frequency of the game performed by the participants.

\section{Frequency of the Game}

In a total of 11 participants, three dropped out. The remaining eight participants were considered for analysis. The analysis shows that only three patients were adherent to Kinect adventure game therapy which was only $38 \%$ which indicates that participants were non-adherent to the Kinect adventure game therapy. From Table 4, it shows that out of 8 participants, three participants adherence was less than 25 $\%$, one participant adherence was between 25 and $50 \%$, one participant adherence was between 51 and $74 \%$ which indicates that these participants were non-adherent to the Kinect adventure game therapy and three participants adherence was more than $75 \%$ which indicates that participants were adherent to Kinect adventure game therapy. The overall adherent percentage to Kinect adventure game therapy was $38 \%$.

\begin{tabular}{|cccc|}
\hline $\begin{array}{c}\text { Adherent } \\
\text { Percentage }\end{array}$ & $\begin{array}{c}\text { Number of } \\
\text { Participants }\end{array}$ & $\begin{array}{c}\text { Range of BESTest } \\
\text { in the Pre-Test }\end{array}$ & $\begin{array}{c}\text { Range of BESTest } \\
\text { in Post-Test }\end{array}$ \\
$25-50 \%$ & $3 / 11$ & $70-85$ & $68-85$ \\
$51-75 \%$ & $1 / 11$ & 63 & 64 \\
$\geq 75$ & $1 / 11$ & 73 & 75 \\
\hline Table 4. Adherence Percentage, No of Participants, the Range of Pre \\
and Post BESTests of Kinect Game Therapy (N = 8)
\end{tabular}

\section{Secondary Outcome Measure - BESTest}

In a total of 11 patients, three dropouts were found. The remaining eight participants were considered for analysis and 6weeks post-test of BESTest score was considered as 
baseline score for game therapy. Baseline and post-test balance scores were analysed using paired t-Test.

\section{$H_{0}$ : mean effect of Post 6-week training and Post 12 weeks \\ (Kinect game therapy was equal. $\mathrm{V} / \mathrm{s}$ \\ $H_{1}$ : mean effect of Post 12 weeks (Kinect game therapy) was greater than Post 6-week training}

\begin{tabular}{|ccc|}
\hline $\mathbf{t}$ & df & P-Value \\
-0.59161 & 7 & 0.7136 \\
\hline Table5. The Difference & between Pre- and Post-Test \\
& Balance Scores of Game Therapy \\
\hline
\end{tabular}

Since the p-value is greater than 0.05 , we do not reject the null hypothesis, meaning the mean effect of Post 6-week training and Post 12 weeks (Kinect game therapy) were equal.

\section{DISCUSSION}

Eleven PD patients were enrolled in the study. Initially, six weeks of home-based balance exercises were given and adherence was measured using the attendance percentage of the sessions completed and it was measured in terms of the frequency of the exercise performed and Kinect game therapy was implemented for another six weeks and adherence was measured using the attendance percentage of the sessions completed and was measured in terms of the frequency of the game performed. The assumption was that patients would be more adherent to game therapy than the home-based balance exercise. However, the results showed that participants were non-adherent to both the interventions, but the adherent percentage for participants in home-based balance exercise was only $9 \%$ which was less when compared to the Kinect game therapy where the adherent percentage was $38 \%$.

The advantage involved in home-based balance exercise was participants could perform the exercise in their home environment which was more comfortable for them.

The disadvantages involved in home-based balance exercise was lack of supervision of the exercise and limited access to exercise resources. The barriers stated by the participants in home-based balance exercises were classified into intrinsic and extrinsic factors. The intrinsic factors included were lack of interest in performing exercises, fear of fall, poor health, fear of doing exercises as an individual and tiredness. Extrinsic factors included bad weather, lack of supervision from a caregiver, personal reasons like tourism, shifting to son / daughter's place, attending family functions, which were the reasons documented by participants. Along with these, home-based exercises have an extraneous disturbance of home which will become the first preference to be occupied, then indulging into the exercise. Moreover, there is no accountability to motivate them to compulsory indulge in the exercise programme. A cross-sectional study was done by Ellis $\mathrm{T}$ to elaborate on the barriers in an exercise program in people with $\mathrm{PD}$, the barriers found where people did not expect to derive benefit from exercise (low outcome expectation), lack of time to perform exercises, poor health, discomfort in the chest, depression, rainy climate, fear of fall, lack of interest, weakness, pain, discomfort with exercise and limited access to exercise resources. ${ }^{16}$ In this study similar kinds of barriers were reported. A systematic review done by Picorelli M, this study elaborated the program characteristics and personal factors, involved in adherence to exercise protocol. The report of the study stated that various personal factors influenced the adherence to drop. The factors stated were reduced mental wellbeing, reduced physical wellbeing and patients who were in the initial stages of depression reported with lower adherent rate to prescribed programs. ${ }^{17}$

Kinect adventure game therapy was implemented for another six weeks after home-based balance exercises and adherence was measured using the attendance percentage of the sessions completed and it was measured in terms of frequency of the game performed. The attendance percentage of Kinect game therapy was $38 \%$ which was still less than 75 $\%$ but the adherence percentage was better compared to the home-based balance exercises. The positives of Kinect game therapy reported by participants were they found games were interesting, fun, enjoyable, motivating and they were themselves involved in the game therapy and their scores were increasing for each session. The game had the interaction between the user and virtual environments. The games involved in the virtual environment with demands of both body(physical) and mind(cognition), it attracted the participants and challenges with visual and auditory feedback, and it stimulated users to exercise as there were rewards and scores which would be motivating. The barriers stated by the participants non-adherent to Kinect game therapy were they felt difficult to come alone, lack of motivation from a caregiver, as there was no caregiver to take them to the rehabilitation centre, poor health, migration and one of the participants stated that he was willing to perform exercise only in-home which could also be the reason for drop out of 3 participants before starting the Kinect game therapy. In this study, all 11 participants underwent a baseline evaluation of BESTest and they had attended six weeks of home-based balance exercises. Before initiating the Kinect adventure game there were three dropouts, with 2 participants who migrated to other cities and one participant who felt difficult to come.

Attendance percentage of home-based balance exercise was analysed week wise up to six weeks. For the first week, the percentage of adherents was $27 \%$, three participants out of eleven adhered to $75 \%$ of attendance. For the second week, the mean percentage of adherents was $27 \%$, three participants out of eleven adhered to $75 \%$ of attendance. There was only $9 \%$ of adherence on the third week, fourth, fifth and sixth week, only one participant out of 11 adhered to $75 \%$ of attendance. It showed a trend that initially participants had more interest to perform exercise after knowing about the benefits of exercise and would have got motivated to do exercise to improve the balance after knowing their score in the BEST test results. As the week progressed, they lost interest and they did not adhere due to the intrinsic and extrinsic factors which were reported by them.

The attendance percentage of Kinect game therapy was analysed week wise up to six weeks. For the first week, the percentage of adherents was $63 \%$, five participants out of eleven adhered to $75 \%$ of attendance. For the second week, the percentage of adherents was $63 \%$, five participants out of eleven adhered to $75 \%$ of attendance. For the third week, the percentage of adherents was $50 \%$, four participants out of eleven adhered to $75 \%$ of attendance. For the fourth week, 
the percentage of adherents was $38 \%$, three participants out of eleven adhered to $75 \%$ of attendance. For the fifth week, the percentage of adherents was $25 \%$, two participants out of eleven adhered to $75 \%$ of attendance.

For the sixth week, the percentage of adherents was 25 $\%$, two participants out of eleven adhered to $75 \%$ of attendance. It shows participants had more interest to play games because it was fun, challenging and motivated by scores and rewards of the game to perform each time better to increase the score and progress from bronze medal to gold / platinum in the virtual environment. The reason for a decrease in the attendance percentage was the factors which were reported by them.

The secondary objective of the study was to evaluate the improvement in balance with home-based balance exercises in PD patients. To check the improvement in BESTest the attendance percentage was categorized into $\leq 25 \%, 26-50$ $\%, 51-75 \%$ and $76-100 \%$. Five participants adhered to 25 $\%$ of attendance.

The best test score range before the intervention was 67 78 and post-intervention the BESTest score improved to the range of $77-79$. There were two participants with $26-50 \%$ adherence. The best test score range before the intervention was 80 - 86 and post-intervention it improved to the range of 85 - 90. There were three participants with 51 - $75 \%$ adherence. The BESTest score range before the intervention was 65 - 74 and post-intervention the score improved to the range of $63-80$.

One participant with adherence of $76-100 \%$. The BESTest score range before the intervention was 82 and postintervention it improved to 92 which was clinically significant with $80 \%$ adherence. For the participants who adhered to 51 - $75 \%$, the difference in BESTest score was 5 for 1 participant and 6 for 2 participants.

This shows that the participant's balance was improved but not significantly. The participants in this group reported that they were satisfied and had an interest to participate in such games. For the participants who adhered to $26-50 \%$ the difference in BESTest score was 4 for 1particpant and 5 for 1 participant. The participants who adhered to $\leq 25 \%$ difference in BESTest score was 1 for 1particpant and the remaining 4 participants there was no difference found in BESTest scores

Eight participants participated in Kinect game therapy. For analysing the BESTest score it was categorized in the same way as home-based therapy. Three participants adhered to $25 \%$ of attendance. The BESTest score range before the intervention was $70-85$, post-intervention the score improved to the range of 68 - 85. There was one participant who adhered to 26 - $50 \%$ of attendance.

The BESTest score before the intervention was 63 and post-intervention it improved to 64 . There was one participant who adhered to $51-75 \%$ of attendance. The BESTest score before the intervention was 73 and postintervention it improved to 75 . Three participants adherence was $76-100 \%$ of attendance (89\%, $100 \%, 100 \%)$.

The BESTest score before the intervention was $77-92$ and post-intervention it was 80 - 95. The participants who adhered $76-100 \%$ the difference in BESTest score was 3 for all three participants. The participants who participated in both home balance therapy and Kinect game therapy were the same.

The improvement in balance was better after post-homebased therapy than Kinect game therapy because the improvement in balance would have reached the maximum and it was maintained when Kinect therapy was continued after home-based therapy.

The procedure of the main study was standardized with a pilot study of 3 PD patients. The exercise intervention was lifetime management and sustainability was needed for PD patients to maintain postural control.

But this sustainability comes only if the therapy was going to be joyful and challenging. The sustainability or adherence to Kinect game therapy could be checked by prescribing it at home.

The Kinect adventure game was not prescribed to each participant's home for checking adherence rather asked the participants to visit the rehabilitation centre because of the cost-effectiveness of Kinect adventure games. Moreover, the participants reported that coming to the rehabilitation centre was difficult for them which may reduce the percentage of adherence to Kinect game therapy. The small samples recruited for the study were very less where we could not find any adherence to both the interventions.

Alternative forms of home exercise prescription should be implicated in order to enhance adherent rates and the home exercise programmes should be prescribed in terms of making instructions to participants simple and less demanding. Social support, providing remainder, reinforcement and family education can be implemented to increase the adherence rate.

\section{CONCLUSIONS}

There was moderate adherence to Kinect adventure game therapy by the participants with Parkinson's disease whereas there was very little adherence to home-based balance exercises. Balance improved after home-based balance exercises whereas the effect was sustained when the participants continued with Kinect adventure therapy.

Data sharing statement provided by the authors is available with the full text of this article at jemds.com.

Financial or other competing interests: None.

Disclosure forms provided by the authors are available with the full text of this article at jemds.com.

\section{REFERENCES}

[1] Pompeu JE, Arduini LA, Botelho AR, et al. Feasibility, safety and outcomes of playing Kinect Adventures! ${ }^{\text {IM }}$ for people with Parkinson's disease: a pilot study. Physiotherapy 2014;100(2):162-8.

[2] Jha PK, Chaudhary N. Epidemiology of Parkinson's disease in Southcentral India-a longitudinal cohort study. IAIM 2017;4(47):8-17.

[3] Galvan A, Wichmann T. Pathophysiology of parkinsonism. Clin Neurophysiol 2008;119(7):1459-74. 
[4] Volpe D, Giantin MG, Maestri R, et al. Comparing the effects of hydrotherapy and land-based therapy on balance in patients with Parkinson's disease: a randomized controlled pilot study. Clin Rehabil 2014;28(12):1210-7.

[5] Borrione P, Tranchita E, Sansone P, et al. Effects of physical activity in Parkinson's disease: a new tool for rehabilitation. World J Methodol 2014;4(3):133-43.

[6] Muzerengi S, Clarke CE. Initial drug treatment in Parkinson's disease. BMJ 2015;351:h4669.

[7] Bacha J, Gomes G, De Freitas T, et al. Effects of kinect adventures games versus conventional physical therapy on postural control in elderly people: a randomized controlled trial. Games Health J 2018;7(1):24-36.

[8] Ribas CG, Alves Da Silva L, Corrêa MR, et al. Effectiveness of exergaming in improving functional balance, fatigue and quality of life in Parkinson's disease: a pilot randomized controlled trial. Parkinsonism Relat Disord 2017;38:13-8.

[9] Afshari M, Yang A, Bega D. Motivators and barriers to exercise in Parkinson's disease. J Parkinsons Dis 2017;7(4):703-11.

[10] Quinn L, Busse M, Khalil H, et al. Client and therapist views on exercise programmes for early-mid stage Parkinson's disease and Huntington's disease. Disabil Rehabil 2010;32(11):917-28.

[11] Clarke C. Parkinson's disease. BMJ 2007;335(7617):4415.
[12] Conradsson D, Löfgren N, Nero H, et al. The effects of highly challenging balance training in elderly with Parkinson's disease: a randomized controlled trial. Neurorehabil Neural Repair 2015;29(9):827-36.

[13] Yitayeh A, Teshome A. The effectiveness of physiotherapy treatment on balance dysfunction and postural instability in persons with Parkinson's disease: a systematic review and meta-analysis. BMC Sports Sci Med Rehabil 2016;8:17.

[14] Shen X, Mak MK. Technology - assisted balance and gait training reduce falls in patients with Parkinson's disease: a randomized controlled trial with 12 - month follow up. Neurorehabil Neural Repair. 2015 Feb;29(2):103 11.

[15] Bacha JMR, Gomes GCV, De Freitas T, et al. Effects of kinect adventures games versus conventional physical therapy on postural control in elderly people: a randomized controlled trial. Games Health J 2018;7(1):24-36.

[16] Ellis T, Cavanaugh JT, Earhart GM, et al. Factors associated with exercise behaviour in people with Parkinson disease. Phys Ther 2011;91(12):1838-48.

[17] Picorelli AM, Pereira LS, Pereira DS, et al. Adherence to exercise programs for older people is influenced by program characteristics and personal factors: a systematic review. J Physiother 2014;60(3):151-6. 